\title{
Pelade et évolution d'une dent de sagesse mandibulaire : présentation d'un cas clinique
}

\author{
Third mandibular molar eruption and alopecia: a case report
}

SIHAM TAISSE, SAMAH CHAJ RI, HAMID RIAHI, IHSANE BENYAHYA

\section{RÉSUMÉ}

Les accidents réflexes dus à l'évolution d'une dent de sagesse mandibulaire surviennent dans une région richement vascularisée et innervée, ce qui expliquerait le développement éventuel de troubles trophiques peladiques. Ce cas concerne un sujet ayant développé une pelade sans aucune cause dentaire apparente, ne répondant pas au traitement dermatologique. Cependant, l'extraction de la dent de sagesse mandibulaire homolatérale, enclavée, a permis la repousse des cheveux. Avec ce cas, on constate comme d'autres auteurs, qu'on ne retrouve pas toujours la concordance entre la dent causale et la topographie de la pelade proposée par Rousseau. Par conséquent, tout patient avec une pelade circonscrite doit faire l'objet d'un examen odontostomatologique approfondi à la recherche d'une éventuelle étiologie dentaire. (Med Buccale Chir Buccale 2005 ; 11 : 181-88). mots clés : pelade, follicule pilo-sébacé, dent de sagesse

\section{SUMMARY}

The reflex accidents to the evolution of the mandibular wisdom tooth are relied on its situation in the very vascularized and innervated regions that lead so to peladic trophic disorders. In this article, we describe a case of alopecia areata without any apparent dental cause with a unsuccess of dermatologic treatment. However, the extraction of heterotopic mandibular wisdom to oth deviated in the alopecia areata side many permit case, we notice as other authors, the non agreement between the causal tooth and the topography of the alopecia areata expressed by Rousseau.

Therefore, any patient with circumscribed alopecia areata must be subject to meticulous odontostomatologic examination in search of possible dental cause. (Med Buccale Chir Buccale $2005 ; 11$ : 181-88).

key words : alopecia areata, pilo-sebaccous follicle, third molar 
médecine buccale chirurgie buccale

L. $11, \mathrm{~N}^{\circ} 3$ 2005

page 182
La pelade d'origine dentaire (POD) appartient aux alopécies circonscrites non cicatricielles et entre dans le cadre des affections bucco-dentaire à distance. Elle peut s'observer à tout âge mais reste plus fréquente chez l'homme jeune [1-3]. La POD n'affecte pas l'état général du patient. Par contre, son impact psychique peut être important puisqu'elle intéresse le cuir chevelu et affecte donc l'image de soi. L'étiopathogénie reste discutée mais dans certains cas la suppression de la cause permet $d$ 'incriminer une réaction réflexe à partir d'épines peladogènes irritatives d'origine dentaire. Le plus souvent, il s'agit d'un foyer infectieux péri-apical, quelquefois c'est l'évolution d'une dent de sagesse mandibulaire qui donne des troubles trophiques peladiques.

Cet article rapporte un cas de pelade sans aucune cause dentaire apparente, ne répondant pas au traitement dermatologique. Cependant, l'extraction d'une dent de sagesse mandibulaire enclavée a permis la repousse des cheveux. Avec ce cas, nous constatons, comme d'autres auteurs, qu'il peut exister une non-concordance entre la dent causale et la topographie de la pelade proposée par Rousseau-Decelle [in1].

\section{OBSER VATION}

Un chirurgien dentiste âgé de 26 ans se présente dans le service d'odontologie chirurgicale pour accompagner un patient. Nous remarquons la présence de deux plaques peladiques sur le cuir chevelu de notre confrère qui porte un calot de façon permanente, depuis plusieurs semaines, afin de dissimuler sa pelade (Fig. 1). II a accepté sans grande conviction la consultation proposée.

L'entretien médical a révélé que le patient ne présentait aucune pathologie générale et qu'il était suivi par un dermatologue pour cette pelade apparue brutalement il y a un an. Ce dernier a posé le diagnostic de pelade psychogène et un traitement médicamenteux initial de 2 mois, à base de «fortifiants » capillaires et de vitaminothérapie, a été instauré. Il a été aussi suggéré à ce confrère, exerçant son activité au sein d'un centre hospitalo-universitaire, d'adopter une hygiène de vie «moins stressante ». Comme il n'y avait aucune amélioration, le confrère a interrompu son traitement au bout de 4 mois. II n'y avait aucun antécédent de soins ou d'extraction dentaires. Les deux plaques peladiques siégent dans la région pariéto-occipitale droite. La première plaque mesurait $3 \mathrm{~cm}$ de diamètre, la

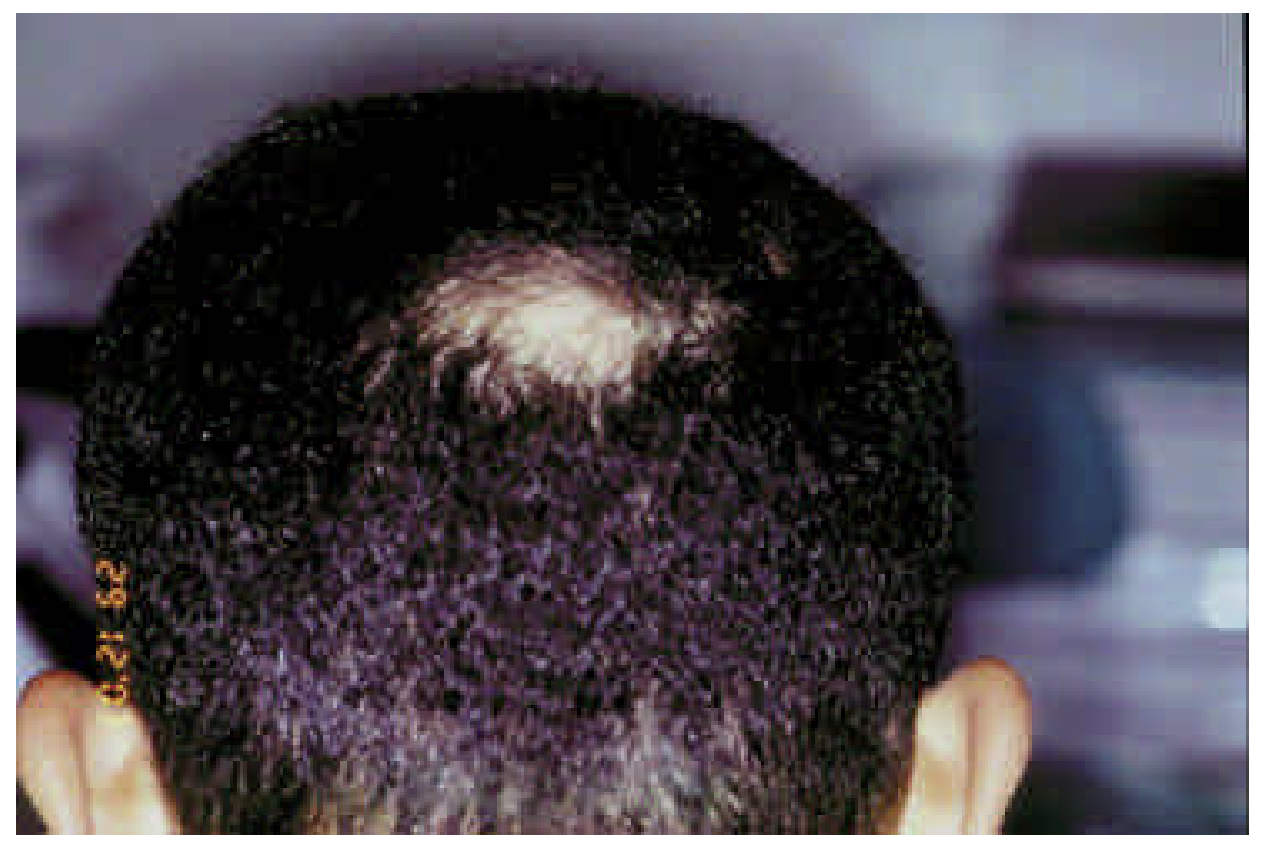

Figure 1 : Pelade du cuir chevelu dans la région pariéto-occipitale droite comportant deux plages : I'une paramédiane, l'autre latérale.

Two peladic areas ont the parieto-occipital calvarium right side 
seconde, plus petite, $1 \mathrm{~cm}$ de diamètre (Fig. 2). Elles sont parfaitement asymptomatiques, propres, non squameuses, lisses à la palpation, avec une pilosité très fine en bordure. L'examen du reste du cuir chevelu, des sourcils et de la barbe ne retrouve aucun autre signe d'alopécie même discrète. L'examen endobuccal ne révèle rien de particulier hormis l'enclavement de la 38 et de la 48. Le son- dage parodontal exclut la présence de poche ou d'inflammation au autour des dents de sagesse mandibulaires (DSM). La radiographie panoramique montre un rapport intime des deux DSM avec le canal alvéolaire inférieur sans aucun signe d'infection (Fig. 3).

Les signes cliniques et radiologiques ne permettent pas d'incriminer l'une ou l'autre des DSM. Comme

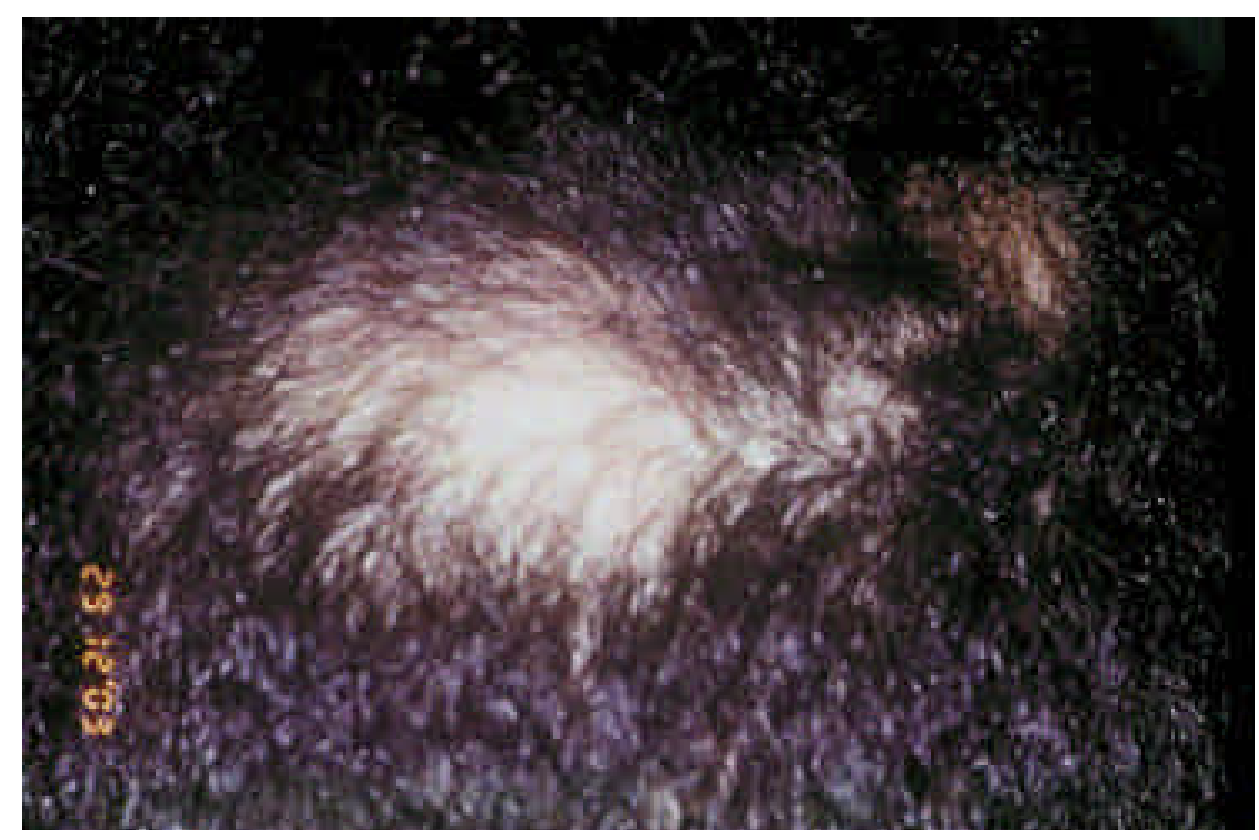

médecin€ buccale chirurgie buccale

VOL. $11, \mathrm{~N}$ 2005

page 183

Figure 2 : La plaque de pelade para-médiane droite mesure $3 \mathrm{~cm}$ de diamètre ; celle qui est latérale $1 \mathrm{~cm}$ de diamètre.

Para-median peladic area have $3 \mathrm{~cm}$ diameter, right peladic area $1 \mathrm{~cm}$ diameter side.

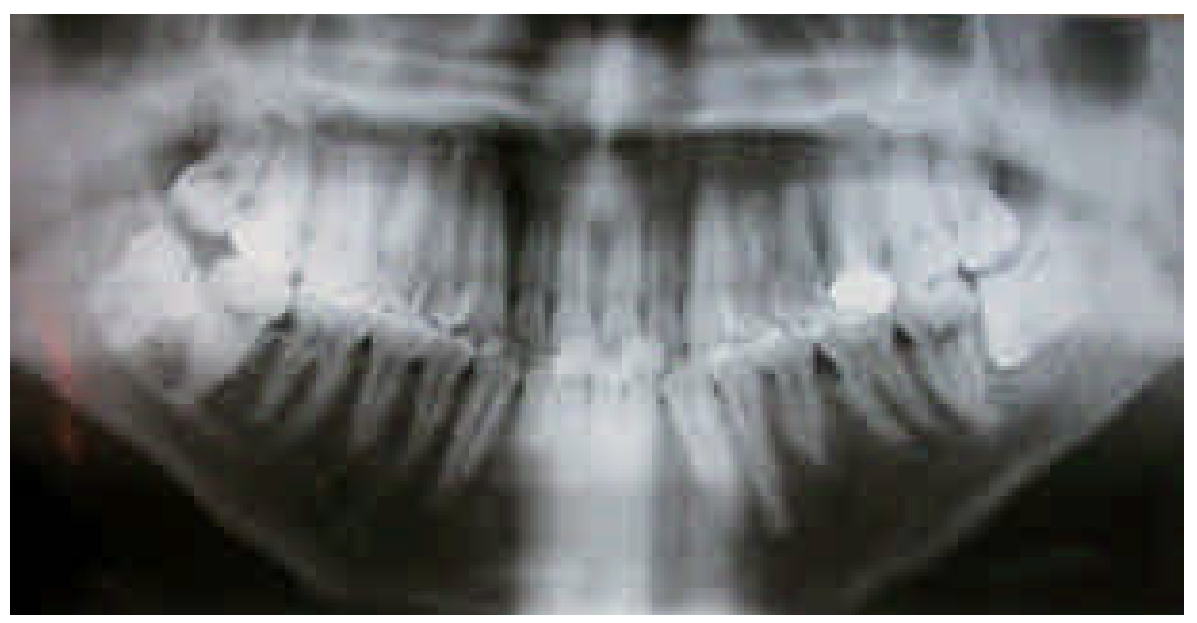

Figure 3 : La radiographie panoramique montre une 38 et une 48 enclavées en position oblique, ayant des rapports intimes avec le canal alvéolaire inférieur.

Panoramic X-ray : 38 and 48 are impacted, near the alveolar duct. 


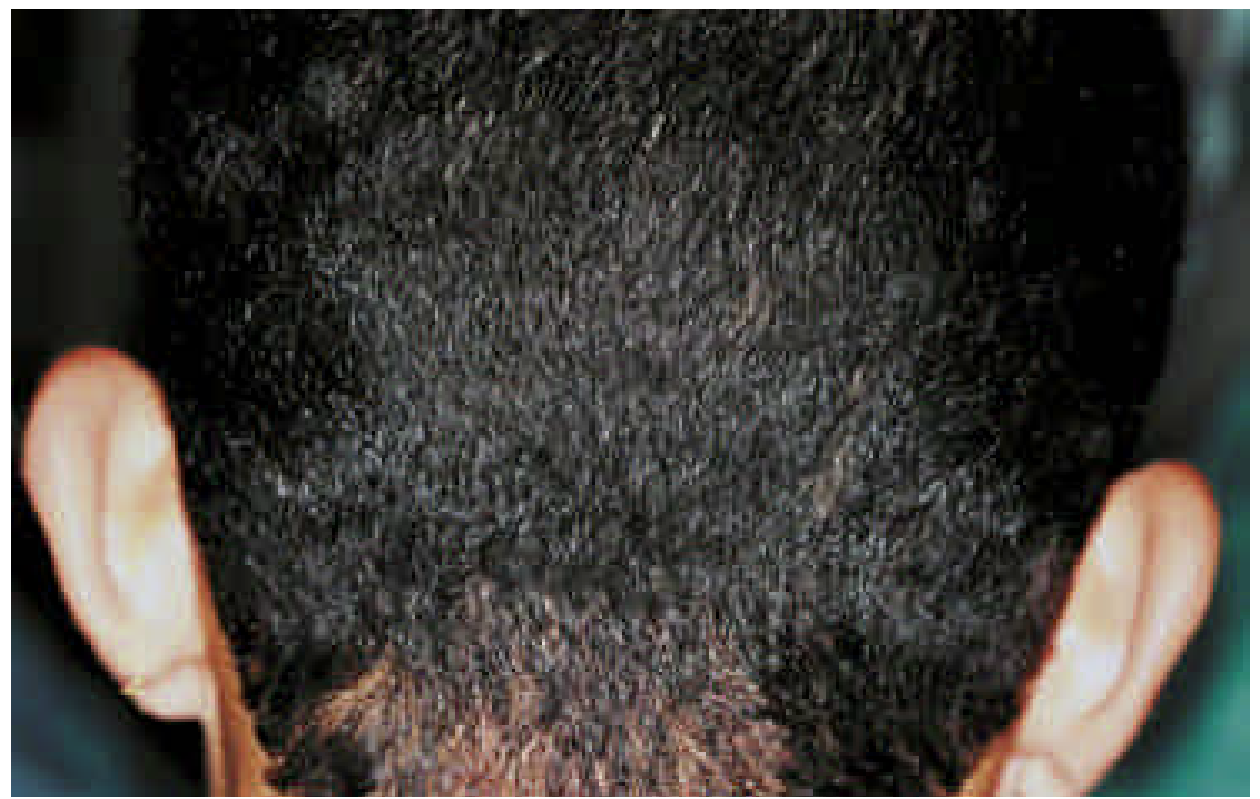

Figure 4 : Disparition totale des deux plages de pelade 4 mois après l'extraction de la 48.

médecine buccale chirurgie buccale

L. $11, \mathrm{~N}^{\circ} 3$ 2005

page 184 Four mounths after avulsion, there is no more alopecia.

les plaques siégent du côté droit, il est apparu logique d'espérer influencer l'évolution de la pelade en pratiquant l'avulsion de la 48, et éventuellement celle de la 38 un peu plus tard. Le confrère a accepté notre plan de traitement et c'est ainsi que l'extraction de la 48 a été programmée sous anesthésie loco-régionale.

Lors des consultations suivantes, une repousse centrifuge notable était remarquée. Les jeunes cheveux étaient fins et peu colorés. A 4 mois, la repousse était complète et le confrère avait renoncé au port permanent du calot (Fig. 4).

\section{COMMENTAIRES}

La POD représente 10 à $18 \%$ des pelades [1-3]; aucune statistique concernant le rôle exact des DSM dans l'apparition de la pelade n'a été retrouvée dans la littérature.

Dans la pelade, on distingue trois fo mes topographiques : la pelade du cuir chevelu, assez souvent d'origine dentaire, se localise préférentiellement dans la région occipitale et elle a tendance à évoluer vers une calvitie totale; la pelade de la barbe ou de la moustache est souvent stable et persistante ; la pelade des cils et des sourcils est exceptionnelle et rarement isolée.

\section{Etiopathogénie}

Elle est très controversée. Plusieurs hypothèses ont été avancées pour expliquer le développement de la pelade. L'hypothèse immunologique s'appuie sur la présence d'un infiltrat lymphocytaire péri-folliculaire et sur l'association possible à une maladie auto-immune (eczéma atopique, vitiligo, anémie pernicieuse...). La pelade semble survenir fréquemment chez des sujets dont l'immunité à médiation cellulaire est diminuée [4-6]. Lors du troisième congrès international de la recherche sur la pelade (Washington DC, 1998), il a été confirmé que les lymphocytes T CD8+ jouent un rôle prépondérant dans la pathogénie de la pelade [7]. Toute irritation bactérienne, mécanique ou chimique, induite par une nécrose pulpaire ou une évolution dentaire, en particulier celle des DSM, peut entraîner des phénomènes inflammatoires avec un infiltrat composé principalement de lymphocytes, mais aussi de plasmocytes ou de macrophages ${ }^{[5,8]}$. La présence de médiateurs immuns communs, intervenant dans la pathogénie des épines irritatives dentaires et de la pelade, pourrait expliquer l'origine dentaire de la pelade [14]. 
L'hypothèse psychosomatique fait jouer au stress psychoaffectif un rôle prépondérant dans le développement de la pelade. L'approche psychosomatique met l'accent sur la personnalité souvent vulnérable des patients développant une pelade [9]. Enfin, l'hypothèse réflexe s'explique par la présence «d'épines irritatives » peladogènes non seulement bucco-dentaires mais aussi oculaires, auriculaires et pharyngées. Le stimulus périphérique, mécanique ou infectieux comme un accident d'évolution de la DSM, est à l'origine d'un arc réflexe trigémino-sympathique qui provoquerait un angiospasme touchant les follicules pileux, entraînant un trouble trophique des cheveux ou des poils d'où leur chute. Comme les follicules pileux restent fonctionnels, les cheveux ou les poils repoussent après la suppression du foyer peladogène responsable [3,10].

Le concept selon lequel la DSM provoquerait des accidents réflexes (troubles trophiques peladiques, musculaires, sécrétoires ou neurologiques) en raison de sa situation dans une région richement vascularisée et innervée, est maintenant controversé. Au terme d'une étude bibliographique exhaustive, l'ANAES a conclu en 1997 «qu'il n'existe aucun argument scientifique prouvant la relation de cause à effet entre la présence $d^{\prime}$ 'une $3^{e}$ molaire mandibulaire en désinclusion et l'une quelconque de ces manifestations » $[11,12]$.

\section{Facteurs favorisants}

Pour de nombreux auteurs, il existe des facteurs favorisant l'apparition de la pelade:

- Un terrain peladique héréditaire, avec transmission autosomique dominante, est retrouvé dans 10 à $20 \%$ des cas. II s'agit d'un élément de mauvais pronostic, avec une tendance évolutive et rebelle de la maladie,

- Un terrain psychique particulier est le plus fréquemment rencontré. II intéresse les personnes timides, anxieuses, neurasthéniques ou agitées,

- Un terrain acquis qui englobe le déséquilib re neurovégétatif, les agénésies pilaires pré ou post-pubériennes, les troubles end ocriniens (le diabète et l'hyperthyroïdie) et les anomalies génétiques telle la trisomie 21 [13].

\section{Diagnostic clinique}

Les plaques peladiques sont généralement isolées ou au nombre de 2 ou 3 , avec quelques centimètres de diamètre. De forme arrondie ou ovalaire, ces plaques sont bien délimitées, glabres, lisses, non squameuses et propres. La chute des cheveux ou des poils est durable mais non définitive, ce qui la différencie de l'alopécie cicatricielle qui reste irréversible, car le tégument est modifié et les follicules pileux détruits [3]. Quelquefois, des cheveux en forme de point d'exclamation, caractéristiques d'une pelade en évolution, sont visibles à la périphérie de la plaque. Dans notre observation, ce type de cheveu n'a pas été remarqué.

Classiquement, les lésions trophiques pilaires apparaissent du même côté que les foyers peladogènes et, en fonction de la localisation de la plaque de pelade, on peut suspecter la dent responsable. Schématiquement, la localisation de la plaque de pelade est d'autant plus postérieure que la dent occupe une position distale sur l'arcade. De plus, les stimuli provenant et des dents mandibulaires et des dents maxillaires intéressent respectivement une zone située en dessus ou en dessous d'une ligne séparant la tête en deux zones : cette ligne va de la commissure labiale à la protubérance occipitale en passant par le conduit auditif externe. Rousseau-Decelle a établi une cartographie des aires peladiques en fonction de la dent causale (Fig. 5). Selon cette cartographie, le siège de la pelade dans le cas rapporté ci-dessus fait suspecter la 17 plutôt que la 48. On peut donc se demander, comme d'autres auteurs [1], si la cartographie de Rousseau-Decelle est toujours fiable?

Par ailleurs, selon les zones de réflexivité de Head, de façon inconstante, une hyperesthésie cutanée d'origine trigéminale précède ou accompagne la plaque de pelade (Fig. 6). Ce signe n'a pas été retrouvé chez notre patient [3].

L'interrogatoire médical revêt une importance particulière puisque l'épisode dentaire peut précéder l'apparition de la pelade de quelques jours à quelques mois. médecin€ buccale chirurgie buccale VOL. $11, \mathrm{~N}$ 2005 page 185 


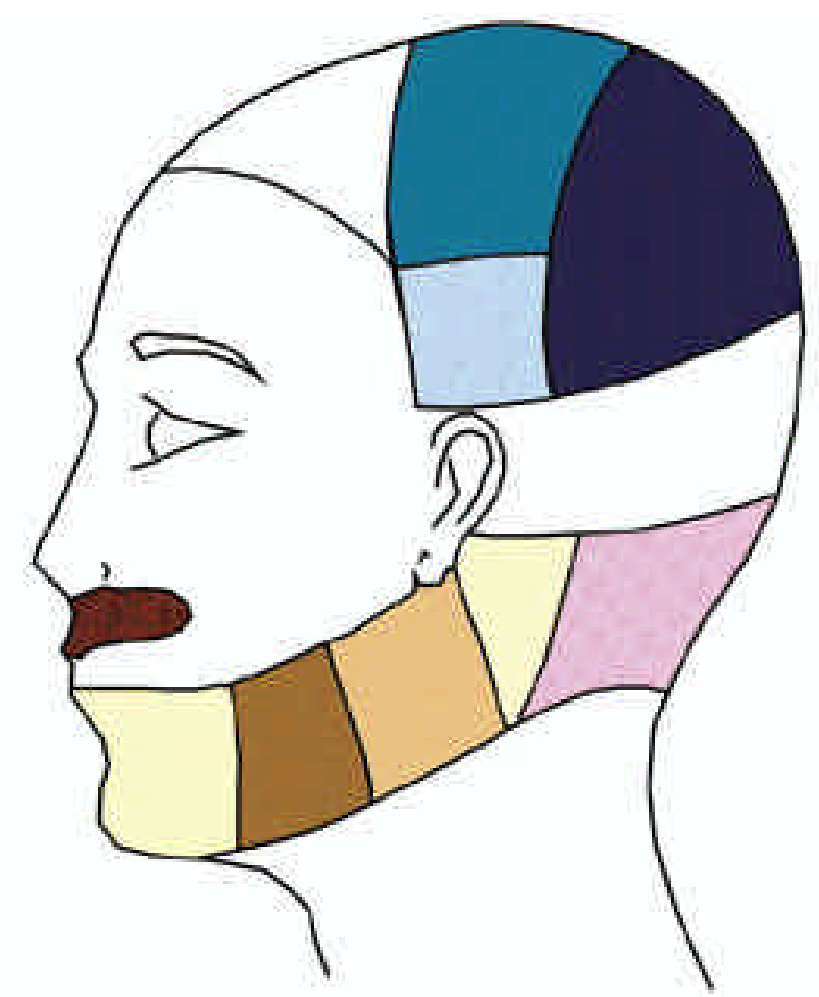

Maxillaire Mandibule Maxilla Mandible

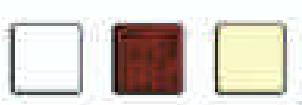

Incisives et canine Prémolaires Premolars
Prémolaires

Premolars

Première molaire

First molar

Deuxième molaire

Second molar

Troisième molaire

Third molar

Figure 5 : Cartographie des lésions trophiques (selon Lesclous P. et Maman L. [12]).

Trophics repercutions areas in (12).

\section{Diagnostic radiologique}

L'imagerie médicale est le complément indispensable de l'examen clinique pour la mise en évidence des foyers peladogènes potentiels. Un cliché panoramique systématique sera éventuellement complété par des radiographies rétroalvéolaires ou occlusaux. Le recours à un examen tomodensitométrique peut être parfois nécessaire.

\section{Traitement}

L'éradication des foyers peladogènes repose sur des traitements conservateurs ou plus radicaux visant à éliminer toute cause d'infection ou de stimulation mécanique des fibres nerveuses [14]. Un traitement endodontique, éventuellement complété par un curetage péri-apical, suffisant dans certains cas. L'orthodontie peut être indiquée pour 


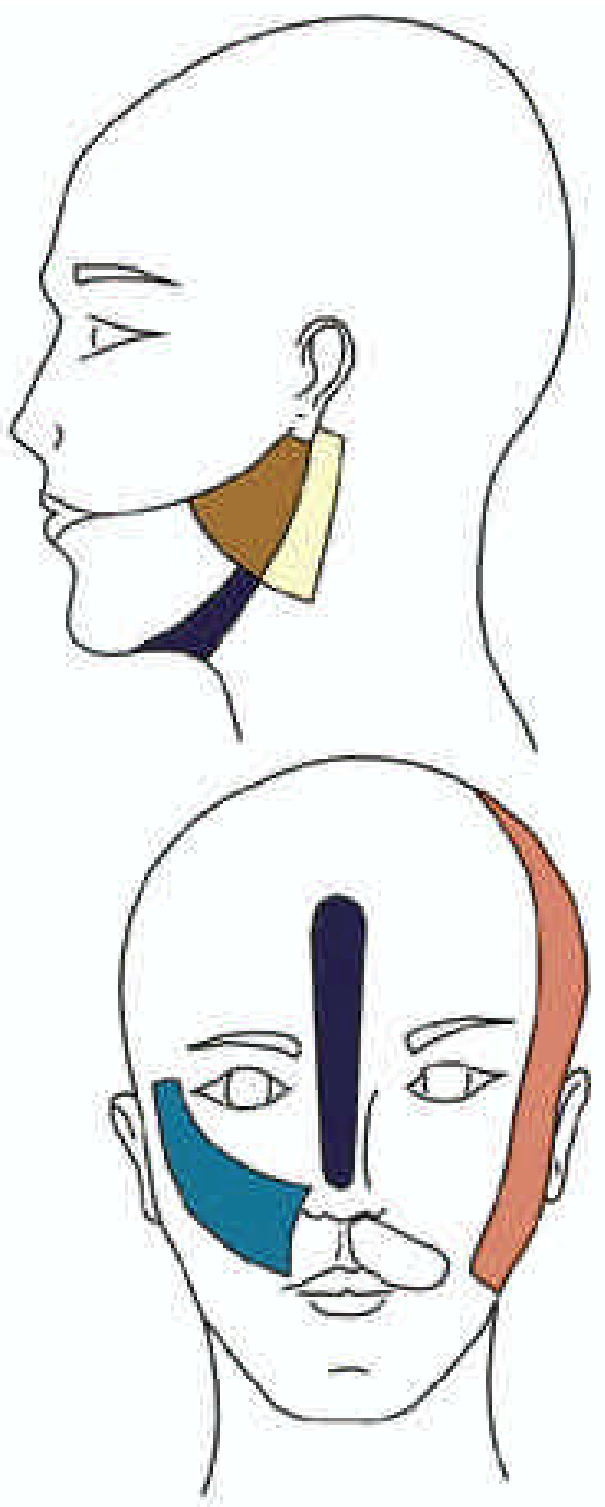

\section{Mandibule Mandible}

Incisives, eanine et première prémolaire Incisars, camine and first promolar

Deuxiäme prémolaire

Secoml premolar

Première et deuxicme molaires

Fint and secoind molars

Troisieme molaire

Thint molor

Maxillaire

Maxilla

Incisives

lencisors

Canine et premičre prcmolaire

Canine and first premolar

médecin

buccale

chirurgie

buccale

VOL. 11, N

\section{Deuxic̉me prémolaire}

Sccond premolar

Première et deuxième molaires

Firat and second molars

Figure 6 :Zones de réflexivité de Head (selon Lesclous P. et Maman L. [12]).

Reflexivity area of Head in (12).

les dents en malposition. L'avulsion chirurgicale est proposée pour les dents incluses ou enclavées, et pour les odontomes.

Une prise en charge dermatologique a le plus souvent été instaurée avant le traitement odontologique. La corticothérapie locale, intra-lésionelle et systémique, est souvent prescrite dans les formes étend ues et récentes [16]. Dans les formes rebelles, on cherche à agir de façon plus spécifique sur l'infiltrat lymphocytaire périfollic ulaire avec une immunothérapie locale. Le diphénylcyclopropenone par exemple favorise la formation des lymphocytes $T$ suppresseurs qui en inhibant la réaction auto-immune, permettent la repousse des cheveux [15] ; ce traitement est réservé au milieu hospitalier. Selon Assouly (2), I'immunothérapie a donné dans certains cas de bons résultats alors que les autres traitements ont échoué. Le minoxidil, initialement commercialisé comme antihypertenseur périphérique, favorise la 
repousse des cheveux car il entraîne une vasodilatation capillaire, empêchant l'atrophie folliculaire. Approuvé par la FDA, il est commercialisé en France en traitement topique à $2 \%$ sous le nom de Regaine ${ }^{\circledR}$. Il est surtout prescrit pour l'alopécie androgénique. La cryothérapie, la vitaminothérapie, la puvathérapie et la prescription d'acides aminés soufrés sont également proposées. Si besoin, une psychothérapie de soutien est conseillée [17-20].

\section{RÉFÉRENCES}

1 - Agence nationale d'Accréditation et d'Evaluation en SANTÉ. Indication et contre-indication de l'avulsion des troisièmes molaires mandibulaires. Recommandations et références médicales, 1997.

2 - ASSOULY Ph. Actualités thérapeutiques dans la prise en charge des pelades. Ann Dematol Venereol 2002 ; $129: 831-6$.

médecine buccale chirurgie buccale
3-Cohen RL, Alves Me, Weiss VC, West DP, ChAmbers DA. Direct effects of minoxidil on epidermal cells in culture. J Invest Dermatol $1984 ; 82: 90-3$.

4 - Colombo S, Keen J A, Brownstein DG, Rhind SM, McGorum BC. Alopecia areata with lymphocytic mural folliculitis affecting the isthmus in a thoroughbred mare. Vet Dermatol $2004 ; 15: 260-5$.

5 - Consoli SG. Psychysme et pathologie du follicule pilosébacé. Rev Prat $1993 ; 18$ : 2370-4

6 - EKOE J M, BURCKHARDT P, RUEDI B. Treatment of hirsutism, acne and alopecia with cyproterone acetate. Dermatologica $1980 ; 160$ : 398-404.

7 - Friedli A, Harms M. Alopecia areata. Ther Umsch 2002 ; 59: 233-7.

8 - KeRn F. Alopecia areata. Immunologic studies and treatment with Prednisone. Arch Dermatol 1973 ; 107: 40712.

9 - Koblenzer CS. Psychotherapy for intractable inflammatory dermatoses. J Am Acad Dermatol 1995 ; 32 : 609-12.

10 - Lesclous P, Maman L. La pelade d'origine dentaire. A propos d'une observation. Inform Dent $1991 ; 39$ : 3443-6.

\section{CONCLUSION}

La relation de cause à effet entre la pelade et l'évolution de la DS M est difficile à démontrer. Seule la régression totale de la pelade permet de confirmer le diagnostic étiologique. L'intérêt d'une collaboration étroite entre le chirurgien dentiste et le dermatologue est un gage de prise en charge correcte de ce type d'affection et devrait permettre de mieux évaluer le rôle exact des foyers peladogènes dentaires.

11 - Lesclous P, Maman L. An unusual case of alopecia areata of dental origin. Oral Surg Oral Med Oral Pathol Oral Radiol Endod $1997 ; 84: 290-2$.

12 - Lesclous P, MAMAn L. La pelade d'origine dentaire: à propos de trois observations cliniques. Méd Buccale Chir Buccale 1996 ; 2 : 31-40.

13 - MC ELWEE KJ .Third Internatioal research workshop on alopecia areata.J Invest Dematol $1999 ; 112$ : 822-4.

14 - Montoya G, Cutando Soriano A, J Imenez P. Alopecia areata of dental origin. Med Oral $2002 ; 7$ : 303-8.

15 - Pericin M, TRÜEB RM. Topical immunotherapy of severe alopecia areata with diphenylcyclopropenone: evaluation of 68 cases. Dermatology $1998 ; 196: 418-21$.

16 - Peron J M. Accidents d'évolution des dents de sagesse. Encycl Méd Chir, Odonto-Stomatol 22-032-E-10, Paris, 2003.

17 - Reychler H, Chausse J M. Pathologie infectieuse d'origine dentaire (pp 1263-88). In : Piette E, Reychler H, éditeurs. Traité de pathologies buccales et maxillofaciales. De Boeck Université, Bruxelles, 1991.

18 - Romoli M, CLAUdiA G. Alopecia areata and homolateral headache due to an impacted superior wisdom tooth. Int J Maxillofac Surg $1987 ; 16: 477-9$.

19 - ToRABINEJ AD M. Mediators of acute and chronic periradicular lesions. Oral Surg Oral Med Oral Pathol 1994 ; $78: 511-21$.

20 - Verma DD, McElwee KJ, Freyschmidt-Paul P, Hoffman R, Fahr A. Treatment of alopecia areata in the DEBR model using Cyclosporin A lipid vesicles. Eur J Dermatol $2004 ; 14$ : 332-8. 